\title{
Mesenchymal stem cells expressing interleukin-18 inhibit breast cancer in a mouse model
}

\author{
XIAOYI LIU ${ }^{*}$, JIANXIA HU $^{2 *}$, YUEYUN LI $^{1}$, WEIHONG CAO $^{1}$, YU WANG ${ }^{1}$, ZHONGLIANG MA $^{1}$ and FUNIAN LI ${ }^{1}$ \\ ${ }^{1}$ Breast Disease Center; ${ }^{2}$ Stem Cell Research Center, The Affiliated Hospital of Qingdao University, \\ Qingdao, Shandong 266003, P.R. China
}

Received July 2, 2015; Accepted January 31, 2017

DOI: $10.3892 /$ ol.2018.8166

\begin{abstract}
Development of an improved breast cancer therapy has been an elusive goal of cancer gene therapy for a long period of time. Human mesenchymal stem cells derived from umbilical cord (hUMSCs) genetically modified with the interleukin (IL)-18 gene (hUMSCs/IL-18) were previously demonstrated to be able to suppress the proliferation, migration and invasion of breast cancer cells in vitro. In the present study, the effect of hUMSCs/IL-18 on breast cancer in a mouse model was investigated. A total of 128 mice were divided into 2 studies (the early-effect study and the late-effect study), with 4 groups in each, including the PBS-, hUMSC-, hUMSC/vector- and hUMSC/IL-18-treated groups. All treatments were injected along with $200 \mu \mathrm{l}$ PBS. Following therapy, the tumor size, histological examination, and expression of lymphocytes, Ki-67, cluster of differentiation 31 and cytokines [interleukin (IL)-18, IL-12, interferon (IFN)- $\gamma$ and TNF- $\alpha$ ] in each group were analyzed. Proliferation of cells (assessed by measuring tumor size and Ki-67 expression) and metastasis, (by determining pulmonary and hepatic metastasis) of breast cancer cells in the hUMSC/IL-18 group were significantly decreased compared with all other groups. hUMSCs/IL-18 suppressed tumor cell proliferation by activating immunocytes and immune cytokines, decreasing the proliferation index of proliferation marker protein Ki-67 of tumor cells and inhibiting tumor angiogenesis. Furthermore, hUMSCs/IL-18 were able to induce a more marked and improved therapeutic effect in the tumor sites, particularly in early tumors. The results of the present study indicate that hUMSCs/IL-18 were able to inhibit the proliferation and metastasis of breast cancer cells
\end{abstract}

Correspondence to: Professor Funian Li, Breast Disease Center, The Affiliated Hospital of Qingdao University, 59 Haier Road, Qingdao, Shandong 266003, P.R. China

E-mail: qdyxyhjx@163.com

${ }^{*}$ Contributed equally

Key words: breast cancer, cancer gene therapy, mesenchymal stem cell, interleukin-18, in vivo study in vivo, possibly leading to an approach for a novel antitumor therapy in breast cancer.

\section{Introduction}

Breast cancer is the most common malignancy in females, accounting for $\sim 1 / 3$ of all types of cancer (1). Despite advances in the detection and treatment of primary and metastatic cancers, and the application of surgery, radiotherapy, chemotherapy, immunotherapy and drug combinations, the mortality rate from cancer remains high, and side effects from the aforementioned combined treatments are severe (2). Therefore, identifying a more effective and less dangerous therapy for cancer is imperative.

Genetically engineered mesenchymal stem cells (MSCs) have been hypothesized to exhibit therapeutic potential in a wide range of human diseases, including cancer. Intravenous injection of MSCs expressing interferon (IFN)- $\beta$ may inhibit the expansion of the pulmonary metastasis of melanoma and breast cancer in mice $(3,4)$, and prolong the survival of mice with glioma xenografts (5). It is well accepted that a critical property of MSCs for cell therapies is their intrinsic homing ability; when infused systemically, MSCs are able to home to the sites of tumor, injury, inflammation and ischemia, although the underlying molecular mechanisms remain unclear $(6,7)$. Previous studies have indicated the role of cytokines and chemokines secreted by target tissues and tumors, including vascular endothelial growth factor, C-C motif chemokine ligand (CCL) 2 and CCL5, in MSC rolling, arrest and transmigration along the endothelium. Following transmigration, MSCs were able to contribute to the antitumor effect by synthesizing the engineered proteins $(8,9)$. It is hypothesized that the innate and acquired immune responses serve crucial roles in the antitumor response, and the interactions between the host immune system and tumor cells have been the subject of intense research over the last decades (10). Cytokines, including transforming growth factor- $\beta$, tumor necrosis factor- $\alpha$ (TNF- $\alpha$ ), interleukin (IL)- 1 , IL-12, IL-18 and IFNs, serve important roles in the immune response $(11,12)$. Alterations in cytokine levels are important parameters that affect the course of disease. IL-18, a more recently described member of the IL-1 cytokine superfamily, is now recognized as an important regulator of innate and acquired immune responses (13). IL-18 is produced by 
macrophages and immature DC early in the acute immune response, and serves an important role in the Th1 response, primarily by its ability to induce IFN- $\gamma$ production by $\mathrm{T}$ cells and natural killer (NK) cells (14). In addition to IFN- $\gamma$, IL-18 also induces granulocyte/macrophage colony-stimulating factor, TNF- $\alpha$ and IL-1 expression, and acts in concert with IL-12 (13,15). Nakata et al (16) demonstrated that IL-18 was able to inhibit osteolytic growth at bone metastatic sites and suppress an early onset of bone metastasis in a breast cancer mouse model. Coskun et al (17) demonstrated that serum IL-18 levels were significantly increased in patients with breast cancer compared with controls. The increase in IL-18 levels was identified to be associated with established clinically used prognostic factors, including tumor size, axillary lymph node involvement and disease stage (18).

Our previous study identified that human MSCs derived from umbilical cord (hUMSCs) genetically modified with the IL-18 gene (hUMSCs/IL-18) were able to significantly decrease the proliferation, migration and invasion of breast cancer cells (MCF-7 and HCC1937 cells) in vitro (19). The underlying molecular mechanism for this suppression of proliferation may be the G1- to S-phase arrest of breast cancer cells induced by hUMSCs/IL-18. The transduced hUMSCs maintained their differentiation potential and pluripotency, and were capable of migration (19). However, limited data exist concerning the presence of IL-18 in human tumors in vivo, therefore, investigation of the effect of hUMSCs/IL-18 on other types of tumor in vivo is warranted.

The aim of the present study was to determine whether hUMSCs/IL-18 were able to inhibit the proliferation and metastasis of breast cancer cells in vivo. A breast cancer mouse model was developed by injecting 4T1 cells subcutaneously (s.c.) into BALB/c mice, and injecting hUMSCs/IL-18 at the early (1 week after injection of 4T1 cells) and late (4 weeks after injection of 4T1 cells) stages of breast cancer, to investigate the safety and effect of hUMSCs/IL-18 on breast cancer progression.

\section{Materials and methods}

Animals. The present study was approved by the Institutional Animal Ethical Committee of Qingdao University (Qingdao, China) and the Ethics Committee of the Affiliated Hospital of Qingdao University. All experimental procedures involving animals were performed in accordance with the Guide for the Care and Use of Laboratory Animals (National Institutes of Health publication no. 80-23, revised 1996) and according to the institutional ethical guidelines for animal experiments. Female BALB/c mice between 6 and 8 weeks of age, with a median weight of $20 \mathrm{~g}$, were purchased from the Laboratory Animal Center of Medical College, Tianjin University (Tianjin, China). All mice were housed in a certified specific pathogen-free animal facility, fed with regular rat chow and maintained under optimal temperature $\left(22-23^{\circ} \mathrm{C}\right)$, light $(12-\mathrm{h}$ light/12-h dark cycle), oxygen, humidity (60\%) and ventilation conditions until sacrifice.

hUMSC/IL-18 preparation. The umbilical cord was obtained from a healthy mother, aged 27 years, following the birth of a healthy term newborn, with no family history of genetic disease, no cancer history, and no presence of hepatitis B virus, hepatitis C virus, human immunodeficiency virus, Epstein-Barr virus, cytomegalovirus or syphilis in serum. Collection of the umbilical cord was approved by the Institutional Medical Research Ethics Committee of Qingdao Maternity Hospital (Shangdong, China). Written informed consent was obtained from the mother 2 weeks prior to delivery.

The preparation of hUMSCs was performed in the laminar flow laboratory, as previously reported (15). A lentivirus construct containing a green fluorescent protein (GFP) gene and mouse IL-18 gene or lentivirus construct containing a GFP gene only (Shanghai GenePharma Co., Ltd., Shanghai, China) was used for the transduction of the hUMSCs. Lentiviruses were added to the medium at room temperature to infect the MSCs at 70 plaque-forming units/cell, and the transfection medium was removed $24 \mathrm{~h}$ later. Effective transduction was confirmed using a human IL-18 ELISA kit (cat. no. KB1138; Shanghai Kaibo Biochemical Reagent Co., Ltd., Shanghai, China) to determine IL-18 levels in the culture supernatant.

Experimental plan. Fig. 1 outlines the plan of the experiment. 4T1 cells were administered as a suspension of $1 \times 10^{6}$ cells in $100 \mu \mathrm{l}$ PBS s.c. into the flank of mice to prepare the breast cancer model. The model was considered successful when the tumor size was $>2 \times 2 \mathrm{~mm}$. In order to investigate the early and the late effects of hUMSC/IL-18 transduction on breast cancer, suspensions of hUMSCs, hUMSCs/vector or hUMSCs/IL-18 in $200 \mu \mathrm{l}$ PBS, or $200 \mu \mathrm{l}$ PBS alone, were injected into each mouse group 1 and 4 weeks after injection of 4T1 cells. Tumor progression and the behavior of the mice were recorded over the course of the experiment.

For the early-effect and late-effect studies, mice with tumors were randomly divided into 4 groups: The PBS group $(\mathrm{n}=16)$, in which mice were injected with $200 \mu \mathrm{l}$ PBS through the lateral tail vein; the hUMSC group $(n=16)$, in which mice were injected with a suspension of $1 \times 10^{6}$ hUMSCs in $200 \mu \mathrm{l}$ PBS; the hUMSC/vector group $(n=16)$, in which mice were injected with a suspension of $1 \times 10^{6} \mathrm{hUMSCs} /$ vector in $200 \mu \mathrm{l}$ PBS; and the hUMSC/IL-18 group ( $\mathrm{n}=16)$, in which mice were injected with a suspension of $1 \times 10^{6}$ hUMSCs/IL-18 in $200 \mu \mathrm{l}$ PBS. Cell suspensions were administered using a $26 \mathrm{G}$ needle via the lateral tail vein.

Assessment of antitumor effect. Tumor dimensions (length, height and width) were measured twice a week using calipers (Dwyer Instruments, Inc., Michigan City, IN, USA) by a researcher blinded to the treatment group, and tumor volume was calculated according to the following formula: Volume=lengthxwidth $2 / 2$. None of the mice had to be sacrificed during the experiment due to tumor ulceration, bleeding or a moribund state with excessive weight loss $>25 \%$ of the initial weight. At the endpoint of the experiment, animals were sacrificed and tumors were excised. To detect IL-18 expression in tissues, and alterations in immune cells and cytokines in vivo, 8 mice from each group were sacrificed 1 week after cell therapy to acquire blood and tissues for analysis. Each experiment was performed at least in triplicate. 


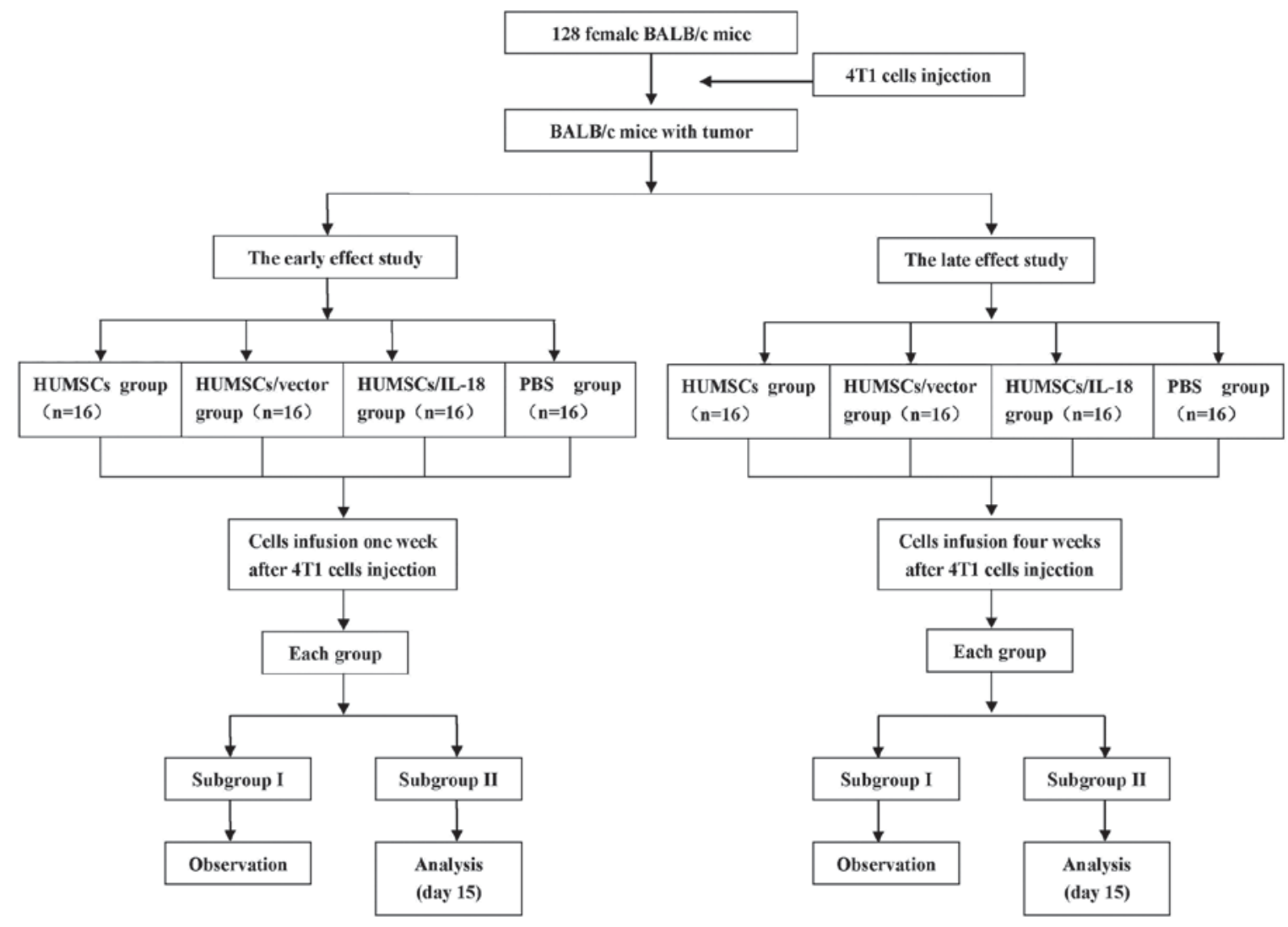

Figure 1. Experimental procedure of the present study. HUMSCs, human mesenchymal stem cells derived from umbilical cord; IL-18, interleukin 18 .

Spleen cell preparation and flow cytometric analysis. Single-cell suspensions were obtained by passing spleens through a 70- $\mu \mathrm{m}$ pore size cell strainer (Falcon; Thermo Fisher Scientific, Inc., Waltham, MA, USA), followed by lysis of erythrocytes with red blood cell lysis buffer (Ortho-Clinical Diagnostics GmbH, Neckargemünd, Germany). The splenocyte suspensions (including lymphocytes and macrophages) were used for flow cytometric analysis. Fluorescein isothiocyanate (FITC)-cluster of differentiation (CD)4, phycoerythrin (PE)-CD8, FITC-CD3 and PE-CD25 monoclonal antibodies (cat. no. CD3-4-8-A, a mix of FITC-CD4, PE-CD8 and FITC-CD3; cat. no. CD3-25-A, a mix of FITC-CD3 and PE-CD25) were added to the splenocyte suspensions and incubated for $30 \mathrm{~min}$ at $4^{\circ} \mathrm{C}$. All antibodies were diluted at 1:200 and purchased from eBioscience, Inc., San Diego, CA, USA. The cells were then washed twice with PBS, fixed with $1 \%$ formaldehyde and analyzed using a FACSCalibur flow cytometer with BD FACStation ${ }^{\mathrm{TM}}$ software version 6.1 (BD Biosciences, Franklin Lakes, NJ, USA).

Histological examination of tumor tissue. Tissue samples from each mouse were divided into thirds. The first third was saved for the analysis of cytokines. Another third was fixed in $4 \%$ formaldehyde solution at room temperature overnight and embedded in paraffin using the conventional method, then cut into $4-\mu \mathrm{m}$-thick sections. Following deparaffinization with xylene (10 min, 2 times) and rehydration with a decreasing gradient concentration of ethanol (100, 90 and $70 \%$ for $5 \mathrm{~min}$ at each concentration), the sections were stained with hematoxylin and eosin for gross histological examination and immunohistochemistry.
The remaining third of the tumor tissues was embedded in OCT medium (Tissue-Tek; Sakura Finetek USA, Inc., Torrance, CA, USA), snap-frozen immediately in liquid nitrogen and stored at $-80^{\circ} \mathrm{C}$ until use. For indirect immunofluorescence analysis, 4- $\mu \mathrm{m}$-thick cryosections were prepared, dried in air, fixed in acetone, rehydrated in PBS and blocked using 10\% goat serum at $37^{\circ} \mathrm{C}$ for $60 \mathrm{~min}$. Sections were incubated with primary antibodies against CD3, CD8, CD16, CD56, CD80 and CD86 (all eBioscience, Inc.) at $4{ }^{\circ} \mathrm{C}$ overnight, followed by three washes in PBS/1\% bovine serum albumin (cat. no. 10437028; Gibco; Thermo Fisher Scientific, Inc.). Subsequently, a FITC-conjugated goat anti-mouse IgG secondary antibody was added (dilution, 1:100; cat. no. 62-6511; Thermo Fisher Scientific, Inc.) for $60 \mathrm{~min}$ to detect primary antibody binding, followed by three washes with PBS. Sections were mounted using Prolong Gold anti-fade mounting medium with DAPI (Invitrogen; Thermo Fisher Scientific, Inc.), examined using a fluorescence microscope and acquired with SPOT software (version 4.0.9; Diagnostic Instruments, Inc., Sterling Heights, MI, USA).

Proliferation index assay of proliferation marker protein $\mathrm{Ki}$-67. An immunohistochemical procedure to detect Ki-67 was performed and results were analyzed using the AxioVision Rel. 4.6 computerized image analysis system assisted by an automatic measurement program (Zeiss AG, Oberkochen, Germany). Following deparaffinization and rehydration, tissue sections were soaked in $3 \% \mathrm{H}_{2} \mathrm{O}_{2}$ at $25^{\circ} \mathrm{C}$ for $10 \mathrm{~min}$, then washed twice in distilled water for $5 \mathrm{~min}$, followed by incubation with an anti-Ki-67 antibody (cat. no. MAB4190; dilution, 
1:300; Sigma-Aldrich; Merck KGaA) at $25^{\circ} \mathrm{C}$ for $60 \mathrm{~min}$. Following washing with PBS, sections were incubated with a peroxidase labeled goat anti-mouse IgG secondary antibody (cat. no. A8924; dilution, 1:300; Sigma-Aldrich; Merck KGaA) at $25^{\circ} \mathrm{C}$ for $45 \mathrm{~min}$, prior to being colored with DAB (Dako REAL $^{\text {TM }}$ EnVision ${ }^{\text {TM }}$ Detection System), counterstained with hematoxylin, dehydrated in a gradient concentration of alcohol and mounted with neutral gum under a light microscope. The stained sections were analyzed at x200 magnification and 10 representative staining fields of each section were analyzed to produce a mean optical density value, which represented the strength of staining signals measured per positive pixel. The mean absorbance data were analyzed to determine statistical differences between groups of tissues.

CD31 examination. Sections were immunostained for CD31, to indicate neovascularization, by incubating them with primary mouse anti-CD31/PECAM-1 monoclonal antibody (dilution, 1:150; cat. no. NB100-1642, Novus Biologicals, LLC, Littleton, $\mathrm{CO}, \mathrm{USA}$ ) and biotinylated goat anti-mouse IgG (dilution, 1:150; cat. no. NBP1-97590; Novus Biologicals, LLC). The sections were then incubated with ExtrAvidin-horseradish peroxidase (Sigma-Aldrich; Merck KGaA, Darmstadt, Germany). Aminoethylcarbazole was used as a chromogenic substrate using an Aminoethylcarbazole Staining kit (Sigma-Aldrich; Merck KGaA). Microphotographs were captured, and 20 random fields of 3 stained sections ( $>4$ fields/section) from each group were observed at $\mathrm{x} 40$ magnification from central healing areas for semiquantitative analysis of microvessel density. Negative control sections were incubated with PBS instead of the primary antibody.

Determination of cytokine levels. Serum samples were obtained by centrifugation $(5,000 \times \mathrm{g}$ for $7 \mathrm{~min}$ at room temperature) of blood (postmortem intracardial puncture) from heparinized mice. A total of 4 cytokines, namely IL-18, IL-12, IFN- $\gamma$ and TNF- $\alpha$, from serum and tumor tissue homogenates were measured using a quantitative sandwich enzyme technique with Quantikine ${ }^{\circledR}$ ELISA kits (BD Biosciences), according to the manufacturer's protocol. The minimum measurable limit of each cytokine was $7.8 \mathrm{pg} / \mathrm{ml}$ for IL-12, $20 \mathrm{pg} / \mathrm{ml}$ for IFN- $\gamma$ and TNF- $\alpha$, and $1.0 \mathrm{pg} / \mathrm{ml}$ for IL-18. Hemolyzed samples were excluded. Samples were assayed in duplicate and the mean absorbance was calculated using a standard curve.

Statistical analysis. Data are presented as the mean \pm standard deviation. Statistical analysis was performed using GraphPad Prism software (version 4.0; GraphPad Software, Inc., La Jolla, CA, USA). Differences among three groups were analyzed by one-way analysis of variance and Bonferroni's post-hoc test. $\mathrm{P}<0.05$ was considered to indicate a statistically significant difference.

\section{Results}

Mouse characteristics. All mice had developed tumors 1 week after injection of 4T1 cells. Prior to cell therapy, no significant differences in the tumor size, diet or vitality of the mice among different groups were identified. Following cell therapy, the diet and vitality of the mice in the hUMSC/IL-18 group were improved compared with the PBS, hUMSC and hUMSC/vector groups.

hUMSCS/IL-18 suppresses tumor proliferation in vivo. The early effect of hUMSCs/IL-18 on breast cancer was evaluated by transduction of hUMSCs/IL-18 1 week after injection of 4T1 cells. Following therapy, proliferation of tumor cells in the mice in the hUMSC/IL-18 group was markedly decreased. There were significant differences in tumor volume between the hUMSC/IL-18 group and the other groups from day 17 $(\mathrm{P}=0.035)$. For the course of the study (day 84$)$, the mean tumor volume in the hUMSC/IL-18 group $\left(0.58 \pm 0.29 \mathrm{~mm}^{3}\right)$ was significantly decreased compared with the other groups $(\mathrm{P}<0.01$; Fig. 2). All mice in the hUMSC/IL-18 group remained alive, whereas only $6 / 8$ mice remained alive in the PBS group, and only $4 / 8$ mice remained alive in each of the hUMSC and hUMSC/vector groups $(\mathrm{P}<0.05)$.

In the late-effect study, proliferation of the tumor cells in the hUMSC/IL-18 group was slightly decreased compared with the other groups following cell therapy. At day 45 of the study, tumor volumes in the PBS, hUMSC and hUMSC/vector groups were increased compared with the hUMSC/IL-18 group ( $\mathrm{P}<0.05$; Fig. 2). Until the end of the study (day 84 ), $6 / 8$ mice remained alive in the hUMSC/IL-18 group, $5 / 8$ mice remained alive in the PBS group, and 4/8 mice remained alive in each of the hUMSC and hUMSC/vector groups ( $\mathrm{P}>0.05)$.

hUMSCS/IL-18 suppresses tumor metastasis. The effect of transduction of hUMSCs/IL-18 on tumor metastasis was evaluated by determining pulmonary and hepatic metastasis in mice with breast cancer. In the early-effect study, following cell therapy, tumor cell metastasis in the hUMSC/IL-18 group was markedly decreased. Until the end of study, 4/8 mice exhibited pulmonary and hepatic metastasis in the hUMSC/IL-18 group, whereas all mice in the PBS, hUMSC and hUMSC/vector groups exhibited pulmonary and hepatic metastasis $(\mathrm{P}=0.021)$. In the late-effect study of hUMSC/IL-18 transduction on tumor metastasis, no positive differences were identified in pulmonary and hepatic metastasis in mice among the 4 groups.

Analysis of tumor tissue. In the early-effect study, using indirect immunofluorescence analysis, lymphocytes that had infiltrated tumors in the hUMSC/IL-18 group were significantly increased compared with the PBS, hUMSC and hUMSC/vector groups. $\mathrm{CD}^{+}$and $\mathrm{CD}^{+} \mathrm{T}$ cells that had infiltrated tumors were significantly increased in the hUMSC/IL-18 group compared with the other groups $(\mathrm{P}<0.01$; Fig. 3$)$. The number of $\mathrm{CD}^{+} 6^{+}$, $\mathrm{CD}^{+} 6^{+}, \mathrm{CD}^{+} 0^{+}$and $\mathrm{CD} 86^{+}$cells in the hUMSC/IL-18 group were significantly increased compared with the other groups $(\mathrm{P}<0.05$; Fig. 3). These results indicated that hUMSCs/IL-18 effectively activated the immunocytes to serve their antitumor roles. In the late-effect study, numbers of $\mathrm{CD}^{+}$and $\mathrm{CD}^{+}$ T cells, and $\mathrm{CD}^{\circ} 6^{+}, \mathrm{CD}^{\circ} 6^{+}, \mathrm{CD} 80^{+}$and $\mathrm{CD} 86^{+} \mathrm{NK}$ cells in the hUMSC/IL-18 group were significantly increased compared with the other groups, but decreased in comparison with those in the hUMSC/IL-18 group in the early-effect study (all $\mathrm{P}<0.05$; Fig. 3).

The levels of cytokines, including IFN- $\gamma$, TNF- $\alpha$, IL-18 and IL-12, were examined in the tumor tissues of the mice 

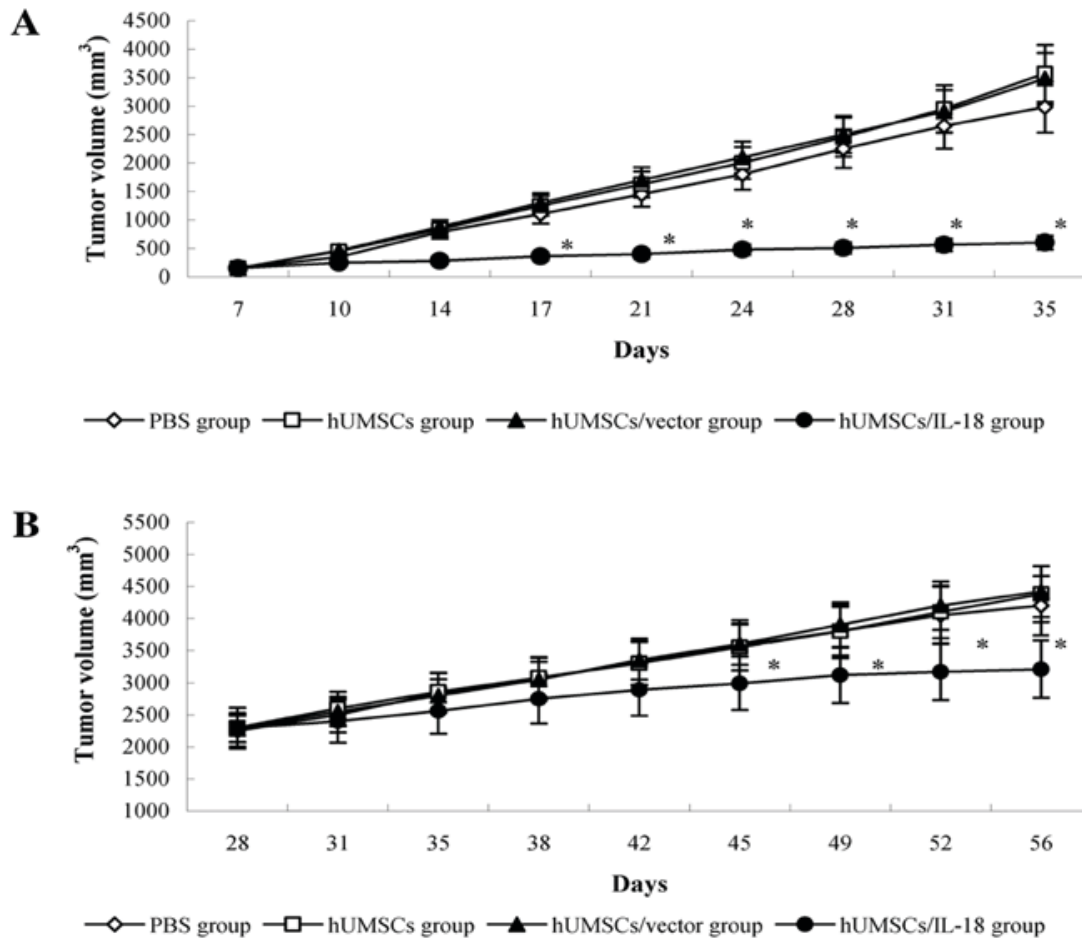

Figure 2. Antitumor effect of cell therapy. Following therapy, 8 mice in each group were monitored for 84 days. (A) In the early-effect study, the tumor volumes of mice in the hUMSC/IL-18 group were significantly decreased compared with the tumor volumes of mice in the PBS, hUMSC and hUMSC/vector groups from day 17 onwards. (B) In the late-effect study, the tumor volumes of mice in the hUMSC/IL-18 group were significantly decreased compared with the tumor volumes of mice in the PBS, hUMSC and hUMSC/vector groups from day 45 onwards. " $\mathrm{P}<0.05$ vs. all other groups. hUMSCs, human mesenchymal stem cells derived from umbilical cord; IL-18, interleukin 18.

A

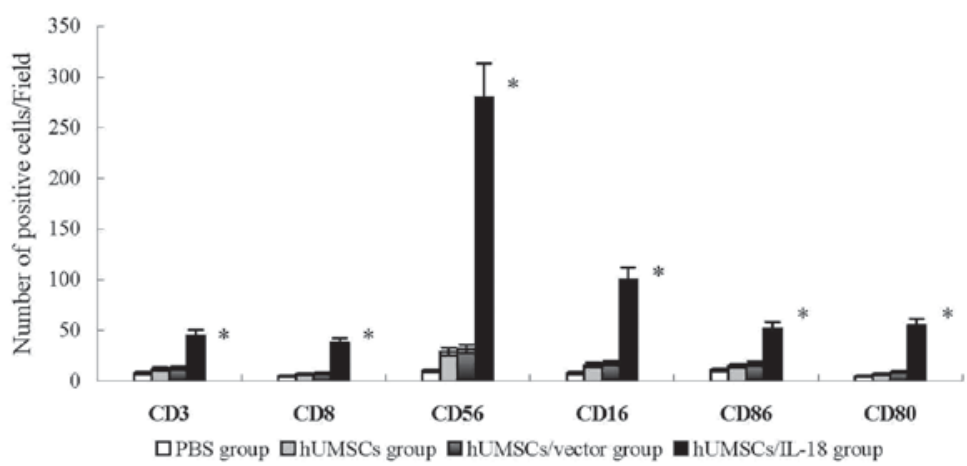

B

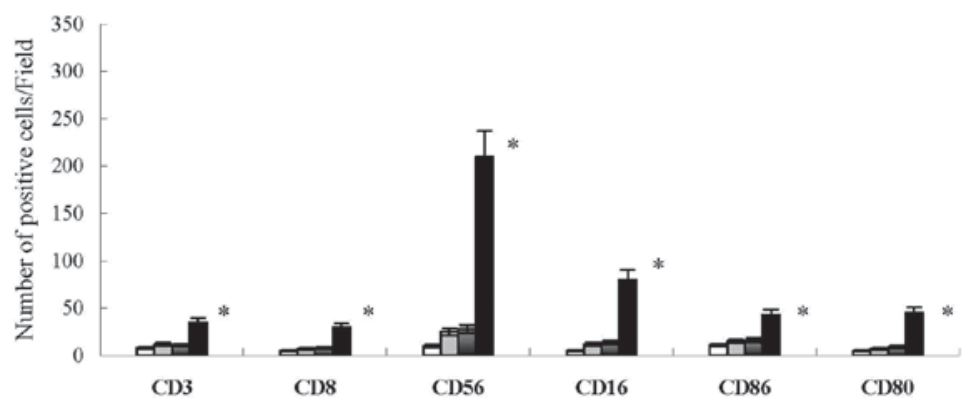

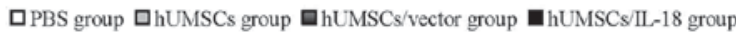

Figure 3. Analysis of lymphocyte infiltration into tumor tissues. Tumor tissues were snap-frozen, and $4-\mu \mathrm{m}$ thick sections were prepared, then stained with fluorescently labeled antibodies. The number of $\mathrm{CD}^{+}$and $\mathrm{CD}^{+} \mathrm{T}$ cells, and $\mathrm{CD} 16^{+}, \mathrm{CD} 56^{+}, \mathrm{CD} 80^{+}$and $\mathrm{CD} 86^{+} \mathrm{NK}$ cells were quantified in 4 sections randomly selected for each group. (A) In the early-effect study, the proportions of $\mathrm{CD}^{+}$and $\mathrm{CD}^{+} \mathrm{T}$ cells, and $\mathrm{CD} 16^{+}, \mathrm{CD}^{2} 6^{+}, \mathrm{CD} 80^{+}$and $\mathrm{CD} 86^{+} \mathrm{NK}$ cells in the hUMSC/IL-18 group were significantly increased compared with the other groups. (B) In the late-effect study, the proportions of $\mathrm{CD} 3^{+}$and $\mathrm{CD} 8^{+} \mathrm{T}$ cells, and $\mathrm{CD}_{16}{ }^{+}, \mathrm{CD}^{2} 6^{+}, \mathrm{CD} 80^{+}$and $\mathrm{CD} 86^{+} \mathrm{NK}$ cells in the hUMSC/IL-18 group were significantly increased compared with those in the other groups, but were decreased compared with those in the hUMSC/IL-18 group in the early-effect study ( $\mathrm{P}=0.039)$. ${ }^{*} \mathrm{P}<0.05$ vs. all other groups. $\mathrm{CD}$, cluster of differentiation; hUMSCs, human mesenchymal stem cells derived from umbilical cord; IL-18, interleukin 18; NK, natural killer. 


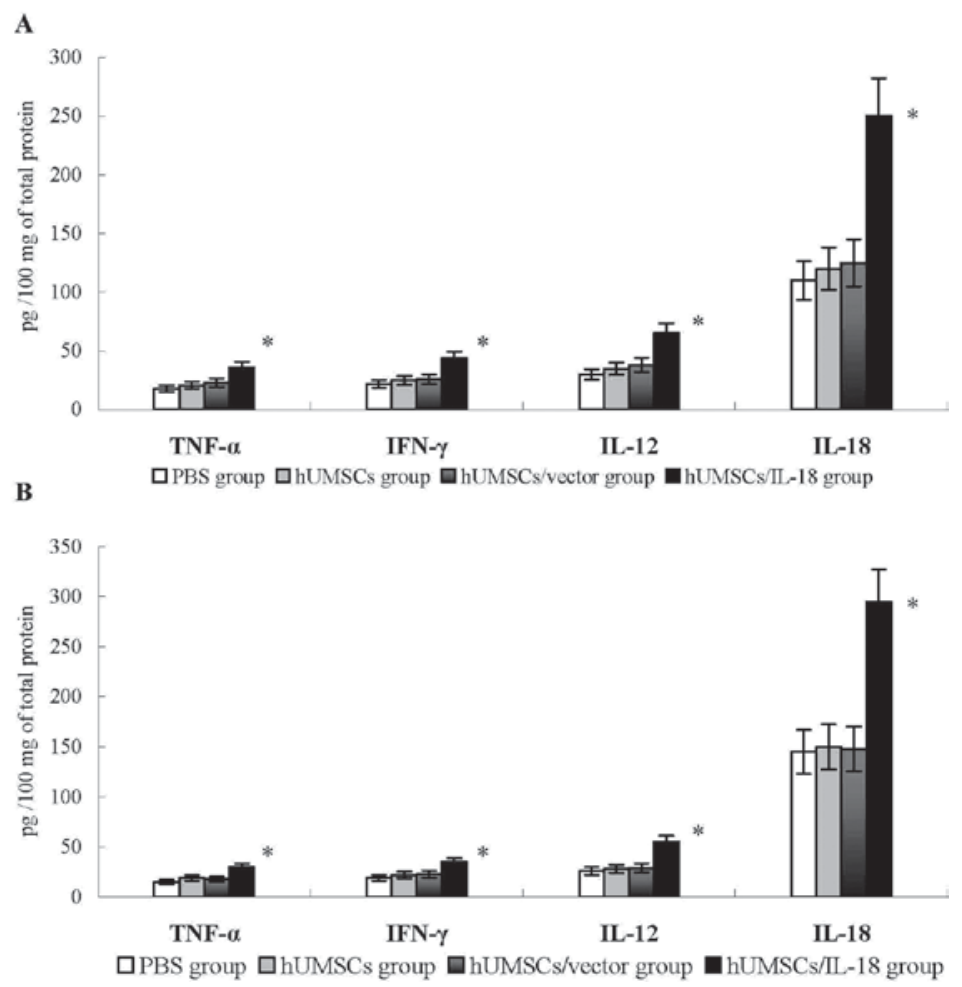

Figure 4. Cytokine levels in tumor tissues. At 1 week after cell therapy, levels of cytokines in tumor tissues, including IFN- $\gamma$, TNF- $\alpha$, IL-18 and IL-12, were determined using ELISA. (A) In the early-effect study, the levels of IFN- $\gamma$, TNF- $\alpha$, IL-18 and IL-12 in the hUMSC/IL- 8 group were significantly increased compared with levels in the PBS, hUMSC and hUMSC/vector groups. (B) In the late-effect study, levels of IFN- $\gamma$, TNF- $\alpha$, IL-18 and IL-12 in hUMSCs/IL-18 group in late infusion study were significantly increased compared with levels in the PBS, hUMSC and hUMSC/vector groups. No significant differences in the levels of cytokines in the hUMSC/IL-18 group between the early-effect and the late-effect study were identified. ${ }^{*} \mathrm{P}<0.05$ vs. all other groups. IFN- $\gamma$, interferon $\gamma$; TNF- $\alpha$, tumor necrosis factor $\alpha$; IL, interleukin; hUMSCs, human mesenchymal stem cells derived from umbilical cord.

1 week after therapy. Compared with the PBS, hUMSC and hUMSC/vector groups, the levels of these cytokines in the hUMSC/IL-18 group were significantly increased $(\mathrm{P}<0.05$; Fig. 4). Similarly, in the late-effect study, levels of IFN- $\gamma$, TNF- $\alpha$, IL-18 and IL-12 in the hUMSC/IL-18 group were significantly increased compared with those in the other groups $(\mathrm{P}<0.05$; Fig. 4). No significant differences in the levels of cytokines in the hUMSC/IL-18 group between the early-effect study and the late-effect study were identified. These results suggested that hUMSCs/IL-18 may induce the release of cytokines to promote the antitumor effect.

Lymphocyte infiltration was greater in the tumors of the hUMSCs/IL-18 group compared with those in the other 3 groups, which indicated a large number of tumor cells and minimal lymphocyte infiltration (Fig. 5). The effect of hUMSCs/IL-18 on tumor angiogenesis was investigated further by examining the expression of CD31 in tumor tissues using immunohistochemistry. The expression of CD31 in the hUMSC and hUMSC/vector groups was significantly increased compared with the PBS and hUMSC/IL-18 groups in the early-effect and the late-effect studies, and expression of CD31 in the hUMSC/IL-18 group was significantly decreased compared with that in the PBS group ( $\mathrm{P}<0.05$; Fig. 5).

In order to evaluate the effect of hUMSCs/IL-18 on the proliferation of breast cancer cells in vivo, the Ki-67 proliferation index was determined using immunohistochemistry. As presented in Fig. 5, the median percentage of proliferating $\mathrm{Ki}-67^{+}$cells within the tumors in the hUMSC/IL-18 group mice was decreased significantly compared with the PBS group [0.38 (range, $0.35-0.67$ ) $\mathrm{Ki}-67^{+}$cells/total cells in the hUMSC/IL-18 group vs. 0.51 (range, $0.42-0.75$ ) in the PBS group, $\mathrm{P}=0.037]$, hUMSC group and hUMSC/vector group [0.67 (range, 0.48-0.90) in the hUMSC group, $\mathrm{P}=0.009$; and 0.63 (range, 0.38-0.83) in the hUMSC/vector group, $\mathrm{P}=0.004]$.

Immune system analysis. In order to compare the difference of levels of cytokines between serum and tumor tissues, the serum levels of cytokines, including IFN- $\gamma$, TNF- $\alpha$, IL-18 and IL-12, were determined in mice 1 week after therapy. Compared with the PBS, hUMSC and hUMSC/vector groups, the serum levels of IL-18 and IL-12 in the hUMSC/IL-18 group were significantly increased $(\mathrm{P}<0.05)$, whereas no significant differences in the levels of IFN- $\gamma$ and TNF- $\alpha$ among the 4 groups were identified (Fig. 6). No significant differences in serum levels of IFN- $\gamma$, TNF- $\alpha$, IL-18 and IL-12 in the hUMSC/IL-18 group were identified between the late-effect and the early-effect study ( $\mathrm{P}>0.05$; Fig. 6).

Spleen lymphocytes of mice in the hUMSC/IL-18 group were markedly activated following therapy (Fig. 7). At 1 week after cell therapy, in the early-effect study, the proportion of $\mathrm{CD}^{+} \mathrm{T}$ cells in the spleen lymphocytes of mice in the PBS, hUMSC, hUMSC/vector and hUMSC/IL-18 groups was $69,71,72$ and $97 \%$, respectively. However, in the late-effect study, the proportions of $\mathrm{CD}^{+} \mathrm{T}$ cells in the spleen lymphocytes of mice in the PBS, hUMSC,hUMSC/vector and hUMSC/IL-18 groups were $52.8,56.6,58.7$ and $85.7 \%$, respectively. Thus, the 
A

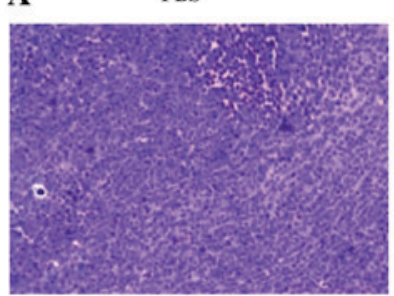

hUMSCs/vector

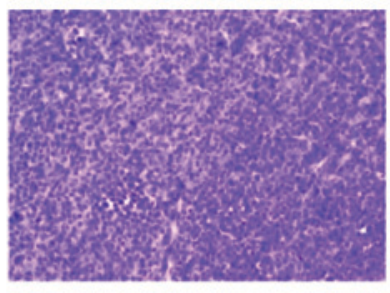

B

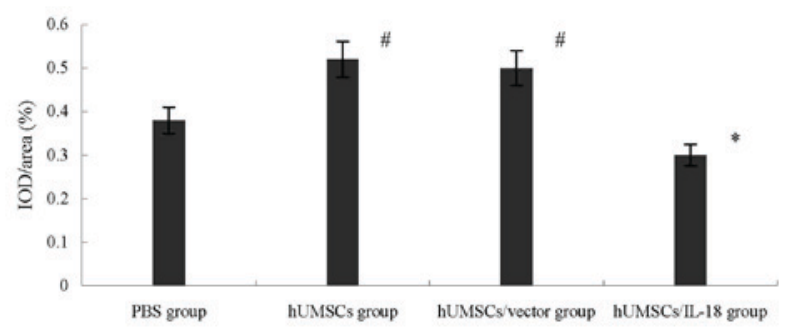

C

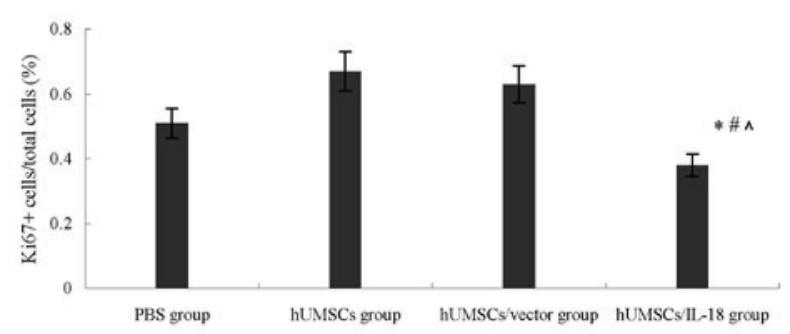

Figure 5.CD31 and Ki-67 proliferation index in tumor tissues. (A) Histological examinations using hematoxylin and eosin staining (magnification, x20). (B) Expression of CD31 in the hUMSC and hUMSC/vector groups was significantly increased compared with that in the PBS and the hUMSC/IL-18 groups $\left({ }^{\#} \mathrm{P}<0.05\right)$, and expression of CD31 in the hUMSC/IL-18 group was significantly decreased compared with that in the PBS group $\left({ }^{*} \mathrm{P}<0.05\right)$ (C) The median proportion of proliferating Ki- $67^{+}$cells within the tumors in the hUMSC/IL-18 group decreased significantly compared with that in the PBS group ( $\mathrm{P}=0.037)$, hUMSC group ( $\left.{ }^{\#} \mathrm{P}=0.009\right)$ and hUMSC/vector group $(\wedge \mathrm{P}=0.004)$. $\mathrm{CD}$, cluster of differentiation; $\mathrm{Ki}-67$, proliferation marker protein Ki-67; hUMSCs, human mesenchymal stem cells derived from umbilical cord; IL-18, interleukin 18; IOD, integrated optical density.

activation of immunocytes in the hUMSC/IL-18 group in the early-effect study was increased compared with that in the late-effect study $(\mathrm{P}=0.039)$. The percentage of $\mathrm{CD}^{+} \mathrm{T}$ cells was markedly increased in the hUMSC/IL-18 group compared with the PBS group ( $\mathrm{P}=0.031$; Fig. 7$)$. Subpopulations of $\mathrm{CD} 4^{+}$ and $\mathrm{CD}^{+} \mathrm{T}$ cells in the 4 groups were analyzed. Compared with the other groups, the percentage of $\mathrm{CD} 3^{+} \mathrm{CD} 8^{+} \mathrm{T}$ cells in the hUMSC/IL-18 group was significantly increased $(\mathrm{P}=0.001$; Fig. 7$)$. For $\mathrm{CD} 4{ }^{+} \mathrm{CD} 25^{+} \mathrm{T}$ cells, the proportion was significantly decreased in the hUMSC/IL-18 group compared with the PBS, hUMSC and hUMSC/vector groups $(\mathrm{P}=0.032$;
Fig. 7). In the late-effect study, the proportion of $\mathrm{CD}^{+} \mathrm{T}$ cells in the hUMSC/IL-18 group was increased slightly compared with the other groups; however, no significant difference was identified ( $\mathrm{P}>0.05$; Fig. 7 ).

Side effects. During the therapy and observation, no abnormality was identified at the sites of injection by macroscopic observations. No acute or chronic side effects were observed in the mice in the hUMSC/IL-18, hUMSC or hUMSC/vector groups compared with the PBS group.

\section{Discussion}

IL-18 serves important roles in cancer progression and metastasis. hUMSCs/IL-18 were able to suppress the proliferation, migration and invasion of MCF-7 cells and HCC1937 cells in vitro in our previous study (19). In the present study, the effect of hUMSCs/IL-18 on breast cancer in a mouse model was further investigated, and it was identified that hUMSCs/IL-18 served antitumor roles in vivo, including the expression of IL-18 protein, the suppression of tumor proliferation and metastasis by activating immunocytes and immune cytokines, decreasing the Ki-67 proliferation index of tumor cells and inhibiting tumor angiogenesis.

It has been demonstrated that MSCs are able to be recruited from the systemic circulation to the stroma of a number of types of growing tumors, including melanoma, glioma, breast cancer and pancreatic cancer (20-23). In tumor sites, MSCs are able to enhance tumor proliferation, invasiveness and the formation of metastases through multiple mechanisms. In addition to differentiating into tumor endothelial cells, pericytes, smooth muscle cells and cancer-associated fibroblasts, MSCs may assist in promoting tumor proliferation by stimulating tumor cells directly in a paracrine fashion $(20,22)$. In the present study, hUMSCs and hUMSCs/vector were able to promote tumor proliferation and metastasis in BALB/c mice with breast cancer. However, the tropism of MSCs to growing tumors also represented a powerful tool to deliver therapeutic substances to these tumors. The transduction of hUMSCs/IL-18 into mice led to significantly reduced tumor proliferation as compared with mice receiving hUMSCs or hUMSCs/vector alone. In the present study, in the hUMSC/IL-18 group, tumor regression was significant from day 17; this result was consistent with the study by Müller et al (24), which demonstrated that IL-18-encoding plasmid DNA exhibited antitumor effects in B16 melanoma and that tumor regression was significant on day 15 of the treatment cycle in the IL-18 group. Furthermore, recombinant murine IL-18 was demonstrated to be effective in B16 melanoma even when the mice were depleted of T cells and NK cells $(25,26)$.

The quantity and quality of immunocyte infiltration into the tumor environment, including the critical balance between effector and regulatory $\mathrm{T}$ cells, have been recognized as vital components of spontaneous and therapy-induced antitumor immune control $(27,28)$. In the present study, following transduction of hUMSCs/IL-18 into mice, the number of immune cells that infiltrated into the tumor site and were activated increased. The proportions of $\mathrm{CD}^{+}$and $\mathrm{CD}^{+} \mathrm{T}$ cells, and $\mathrm{CD}^{+} 6^{+}, \mathrm{CD}^{2} 6^{+}$, $\mathrm{CD}^{+} 0^{+}$and $\mathrm{CD}^{+} 6^{+} \mathrm{NK}$ cells in the hUMSC/IL-18 group were increased compared with the other groups. These results indicated that hUMSCs/IL-18 were able to serve antitumor 

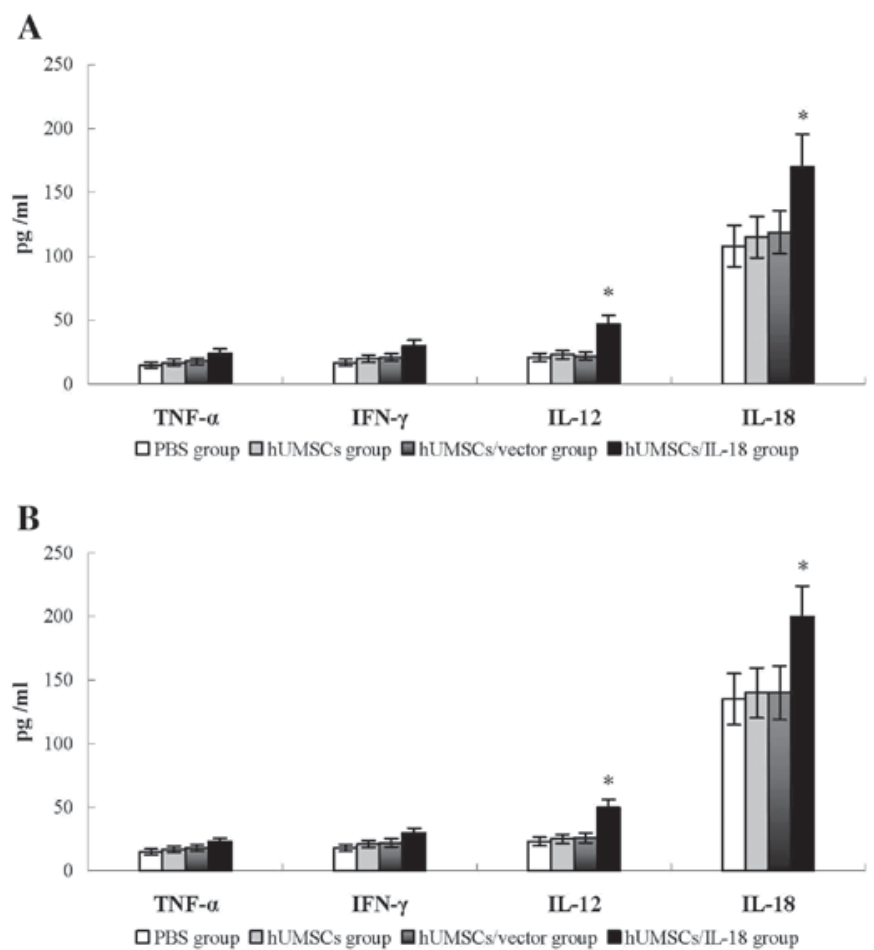

Figure 6. Cytokine levels in peripheral blood. At 1 week after cell therapy, serum levels of cytokines, including IFN- $\gamma$, TNF- $\alpha$, IL-18 and IL-12, were determined using ELISA. (A) In the early-effect study, the serum levels of IL-18 and IL-12, but not of TNF- $\alpha$ or IFN- $\gamma$, in the hUMSC/IL-18 group were significantly increased compared with levels in the PBS, hUMSC and hUMSC/vector groups. (B) In the late-effect study, the serum levels of IL-18 and IL-12, but not of TNF- $\alpha$ or IFN- $\gamma$, in the hUMSC/IL-18 group were significantly increased compared with levels in the PBS, hUMSC and hUMSC/vector groups. No significant differences in serum levels of IFN- $\gamma$, TNF- $\alpha$, IL-18 and IL-12 in the hUMSC/IL-18 group between the late-effect and the early-effect study were identified. "P<0.05, vs. all other groups. IFN- $\gamma$, interferon $\gamma$; TNF- $\alpha$, tumor necrosis factor $\alpha$; IL, interleukin; hUMSCs, human mesenchymal stem cells derived from umbilical cord.

PBS

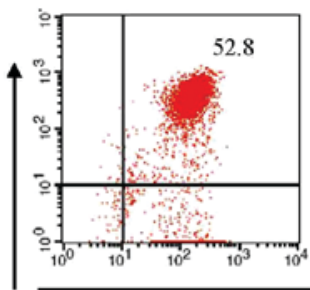

CD3 FITC

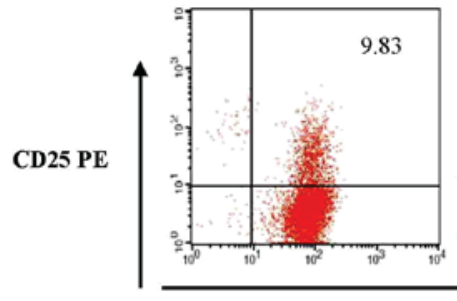

hUMSCs

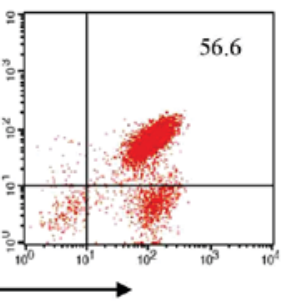

56.6

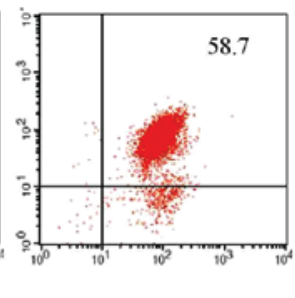

HUMSCs/vector

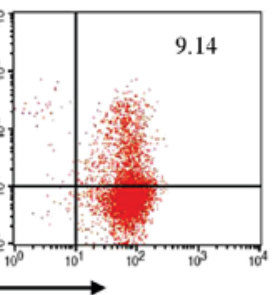

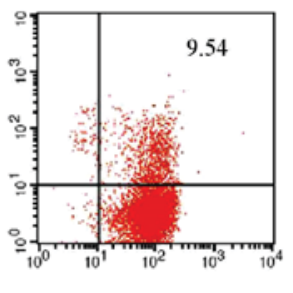

HUMSCs/IL-18
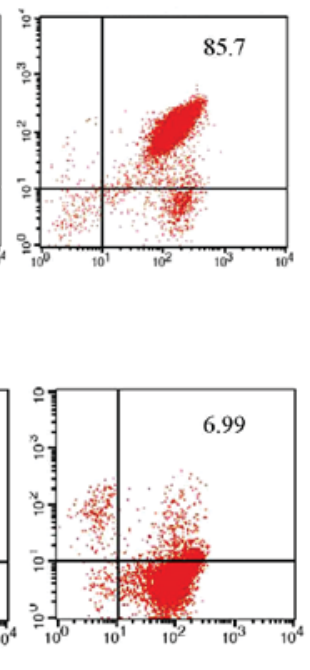

CD4 FITC

Figure 7. Flow cytometric analysis of spleen lymphocyte subpopulations. At 1 week after cell therapy, 8 mice in each group were sacrificed to collect spleen lymphocytes for analysis. The proportion of $\mathrm{CD}^{+} \mathrm{CD}^{+} \mathrm{T}$ cells in the hUMSC/IL-18 group was significantly increased compared with the PBS, hUMSC and hUMSC/vector groups ( $\mathrm{P}=0.007,0.023$ and 0.039 , respectively). Conversely, the proportion of $\mathrm{CD} 4^{+} \mathrm{CD} 25^{+} \mathrm{T}$ cells in the hUMSC/IL-18 group was significantly decreased compared with the PBS, hUMSC and hUMSC/vector groups ( $\mathrm{P}=0.009,0.035$ and 0.021 , respectively). CD, cluster of differentiation; hUMSCs, human mesenchymal stem cells derived from umbilical cord; IL-18, interleukin 18; PE, phyocerythrin; FITC, fluorescein isothiocyanate.

roles by stimulating the cytotoxic activity of NK and T cells. Furthermore, levels of cytokines, including IFN- $\gamma, \mathrm{TNF}-\alpha$,
IL-18 and IL-12, in tumor tissues were also increased in the hUMSC/IL-18 group. IL-18 alone or in synergy with other 
cytokines, including IL-12, modulated the differentiation, proliferation, function and survival of immune cells $(29,30)$. NK cells activated by IL-18 were able to induce the activation of dendritic cells (DCs) and potentiate DC-mediated induction of tumor-specific Th1 and cytotoxic T lymphocyte adaptive immune responses through an IFN- $\gamma$ - and TNF- $\alpha$-dependent mechanism $(31,32)$. NK cells may act as the inducers of local immune cell accumulation, promoting the CCR5-dependent attraction of immature DCs and driving subsequent DC production of the effector $\mathrm{CD}^{+} \mathrm{T}$ cell-recruiting chemokines C-X-C motif chemokine ligand (CXCL)9, CXCL10 and CCL5, in cells isolated from the blood of healthy donors and in tumor-associated cells isolated from the malignant ascites of patients with advanced (stage III-IV) cancer (33).

It was previously reported that IL-18 was able to inhibit the proliferation of tumor cells by inhibiting tumor angiogenesis (34). CD31 is considered a marker that indicates the degree of tumor angiogenesis (35). In the present study, immunohistochemical staining of CD31 indicated that treatment with hUMSCs/IL-18 resulted in reduced vessel formation compared with treatment with hUMSCs or hUMSCs/vector. Furthermore, the treatment of mice with hUMSCs/IL-18 led to a significantly decreased Ki-67 proliferation index in tumors compared with that in mice receiving hUMSCs or hUMSCs/vector alone. In our previous in vitro study (19), it was demonstrated that hUMSCs/IL-18 were able to induce the G1- to S-phase arrest of breast cancer cells. These results indicated that hUMSCs/IL-18 were able to suppress tumor proliferation by inhibiting tumor angiogenesis and decreasing $\mathrm{Ki}-67$ proliferation indices in tumors.

As a main cause of cancer mortality, metastasis is a complex process involving a range of overlapping events, including cancer cell attachment to the extracellular matrix, and cell invasion and migration (36). Yamada et al (37) demonstrated that IL-18 suppressed the pulmonary metastasis of osteosarcoma cells, independently of T and NK cells, by inducing one or more host factors that suppressed cell mobility to inhibit the settlement of osteosarcoma cells in the lung. Furthermore, previous studies have demonstrated that increased IL-18 serum levels accompany tumor progression and have a negative prognostic impact on patients with cancer (38-40). In the present early-effect study, transduction of hUMSCs/IL-18 into mice significantly suppressed the pulmonary metastasis and hepatic metastasis of breast cancer, whereas in the late-effect study, the suppressing effect on tumor metastasis was not evident. The underlying molecular mechanisms remain unclear; however, it has been demonstrated previously that IL-18 alone accelerated tumor progression in the absence of Th1-like cytokines, in part through cell autonomous effects on cancer and endothelial cells (41). In addition, low doses of IL-18 were able to mediate immunosuppression on the NK cell arm of immunity $(42,43)$.

In the present study, the difference between cytokines and immunocytes that had infiltrated into tumor tissues and into the systemic circulation was compared. The levels of cytokines and immunocytes that had infiltrated into tumor tissues were increased compared with those that had infiltrated into the systemic circulation. The levels of cytokines in tumor tissues were increased $\sim 1.5$-fold compared with those in the systemic circulation. This result may be due to the locally increased concentration of IL-18 and partial release into the bloodstream, and also indicated that increased numbers of hUMSCs/IL-18 migrated to the tumor sites to serve this role. The early effect and the late effect of hUMSCs/IL-18 on breast cancer were also investigated. For the early effect, hUMSCs/IL-18 significantly suppressed tumor proliferation and tumor metastasis, whereas for the late effect, the therapeutic effect was not as marked as the early effect, particularly for tumor metastasis. This result indicated that the early effect of treatment offered an improvement over the late effect of treatment.

The results of the present study identified that hUMSCs/IL-18 were able to inhibit the proliferation and metastasis of tumor cells in a mouse model of breast cancer. hUMSCs/IL-18 suppressed the proliferation of tumor cells by activating immunocytes and immune cytokines, decreasing the $\mathrm{Ki}-67$ proliferation index of tumor cells and inhibiting tumor angiogenesis. Furthermore, hUMSCs/IL-18 were able to induce an improved therapeutic effect in the tumor sites, particularly in early tumors. However, the present study represents a preliminary study, and further investigations are required to identify how to increase the expression of IL-18, how to determine the optimal number and duration of cell infusion for cancer therapy, and how to combine cell therapy with chemotherapy or radiotherapy, particularly for advanced tumors.

\section{Acknowledgements}

The present study was supported by the Department of Central Laboratory, The Affiliated Hospital of Qingdao University, and by the Shandong Provincial Natural Science Foundation (grant no. 2013ZRB01426).

\section{References}

1. Schrijver WAME, Schuurman K, van Rossum A; Dutch Distant Breast Cancer Metastases Consortium, Peeters T, Ter Hoeve N, Zwart W, van Diest PJ and Moelans CB: Loss of steroid hormone receptors is common in malignant pleural and peritoneal effusions of breast cancer patients treated with endocrine therapy. Oncotarget 8: 55550-55561, 2017.

2. Zeidan AM, Long JB, Wang R, Hu X, Yu JB, Huntington SF, Abel GA, Mougalian SS, Podoltsev NA, Gore SD, et al: Risk of myeloid neoplasms after radiotherapy among older women with localized breast cancer: A population-based study. PLoS One 12: e0184747, 2017.

3. Ahn JO, Lee HW, Seo KW, Kang SK, Ra JC and Youn HY: Anti-tumor effect of adipose tissue derived-mesenchymal stem cells expressing interferon- $\beta$ and treatment with cisplatin in a xenograft mouse model for canine melanoma. PLoS One 8: e74897, 2013

4. Ryu H, Oh JE, Rhee KJ, Baik SK, Kim J, Kang SJ, Sohn JH, Choi E, Shin HC, Kim YM, et al: Adipose tissue-derived mesenchymal stem cells cultured at high density express IFN- $\beta$ and suppress the growth of MCF-7 human breast cancer cells. Cancer Lett 352: 220-227, 2014.

5. Lee DH, Ahn Y, Kim SU, Wang KC, Cho BK, Phi JH, Park IH, Black PM, Carroll RS, Lee J and Kim SK: Targeting rat brainstem glioma using human neural stem cells and human mesenchymal stem cells. Clin Cancer Res 15: 4925-4934, 2009.

6. Knoop K, Schwenk N, Dolp P, Willhauck MJ, Zischek C, Zach C, Hacker M, Göke B, Wagner E, Nelson PJ and Spitzweg C: Stromal targeting of sodium iodide symporter using mesenchymal stem cells allows enhanced imaging and therapy of hepatocellular carcinoma. Hum Gene Ther 24: 306-316, 2013.

7. Payne NL, Sun G, McDonald C, Layton D, Moussa L, Emerson-Webber A, Veron N, Siatskas C, Herszfeld D, Price J and Bernard CC: Distinct immunomodulatory and migratory mechanisms underpin the therapeutic potential of human mesenchymal stem cells in autoimmune demyelination. Cell Transplant 22: 1409-1425, 2013. 
8. Molloy AP, Martin FT, Dwyer RM, Griffin TP, Murphy M, Barry FP, O'Brien T and Kerin MJ: Mesenchymal stem cell secretion of chemokines during differentiation into osteoblasts, and their potential role in mediating interactions with breast cancer cells. Int $\mathrm{J}$ Cancer 124: 326-332, 2009.

9. Dwyer RM, Potter-Beirne SM, Harrington KA, Lowery AJ, Hennessy E, Murphy JM, Barry FP, O'Brien T and Kerin MJ: Monocyte chemotactic protein-1 secreted by primary breast tumors stimulates migration of mesenchymal stem cells. Clin Cancer Res 13: 5020-5027, 2007.

10. Macho-Fernandez E, Cruz LJ, Ghinnagow R, Fontaine J, Bialecki E, Frisch B, Trottein F and Faveeuw C: Targeted delivery of $\alpha$-galactosylceramide to CD8 $\alpha+$ dendritic cells optimizes type I NKT cell-based antitumor responses. J Immunol 193: 961-969, 2014.

11. Keyhani A, Riazi-Rad F, Pakzad SR and Ajdary S: Human polymorphonuclear leukocytes produce cytokines in response to Leishmania major promastigotes. APMIS 122: 891-897, 2014.

12. Sun H, Sun C and Xiao W: Expression regulation of co-inhibitory molecules on human natural killer cells in response to cytokine stimulations. Cytokine 65: 33-41, 2014

13. Lim HX, Hong HJ, Cho D and Kim TS: IL-18 enhances immunosuppressive responses by promoting differentiation into monocytic myeloid-derived suppressor cells. J Immunol 193: 5453-5460, 2014.

14. Jarry A, Malard F, Bou-Hanna C, Meurette G, Mohty M, Mosnier JF, Laboisse CL and Bossard C: Interferon-alpha promotes Th1 response and epithelial apoptosis via inflammasome activation in human intestinal mucosa. Cell Mol Gastroenterol Hepatol 3: 72-81, 2016.

15. Serti E, Werner JM, Chattergoon M, Cox AL, Lohmann V and Rehermann B: Monocytes activate natural killer cells via inflammasome-induced interleukin 18 in response to hepatitis $\mathrm{C}$ virus replication. Gastroenterology 147: 209-220.e3, 2014.

16. Nakata A,TsujimuraT,Sugihara A,OkamuraH,IwasakiT,ShinkaiK, Iwata N, Kakishita E, Akedo H and Terada N: Inhibition by interleukin 18 of osteolytic bone metastasis by human breast cancer cells. Anticancer Res 19: 4131-4138, 1999.

17. Coskun U, Gunel N, Sancak B, Onuk E, Bayram M and Cihan A: Effect of tamoxifen on serum IL-18, vascular endothelial growth factor and nitric oxide activities in breast carcinoma patients. Clin Exp Immunol 137: 546-551, 2004.

18. Ye ZB, Ma T, Li H, Jin XL and Xu HM: Expression and significance of intratumoral interleukin-12 and interleukin-18 in human gastric carcinoma. World J Gastroenterol 13: 1747-1751, 2007.

19. Liu X, Hu J, Sun S, Li F, Cao W, Wang YU, Ma Z and Yu Z: Mesenchymal stem cells expressing interleukin-18 suppress breast cancer cells in vitro. Exp Ther Med 9: 1192-1200, 2015.

20. Doucette T, Rao G, Yang Y, Gumin J, Shinojima N, Bekele BN, Qiao W, Zhang W and Lang FF: Mesenchymal stem cells display tumor-specific tropism in an RCAS/Ntv-a glioma model. Neoplasia 13: 716-725, 2011.

21. Sage EK, Kolluri KK, McNulty K, Lourenco Sda S, Kalber TL, Ordidge KL, Davies D, Gary Lee YC, Giangreco A and Janes SM: Systemic but not topical TRAIL-expressing mesenchymal stem cells reduce tumour growth in malignant mesothelioma. Thorax 69: 638-647, 2014.

22. Esposito M and Kang Y: Targeting tumor-stromal interactions in bone metastasis. Pharmacol Ther 141: 222-233, 2014.

23. Moniri MR, Sun XY, Rayat J, Dai D, Ao Z, He Z, Verchere CB, Dai LJ and Warnock GL: TRAIL-engineered pancreas-derived mesenchymal stem cells: Characterization and cytotoxic effects on pancreatic cancer cells. Cancer Gene Ther 19: 652-658, 2012.

24. Müller J,Feige K, Wunderlin P, Hödl A, Meli ML, Seltenhammer M, Grest P, Nicolson L, Schelling C and Heinzerling LM: Double-blind placebo-controlled study with interleukin-18 and interleukin-12-encoding plasmid DNA shows antitumor effect in metastatic melanoma in gray horses. J Immunother 34: 58-64, 2011.

25. Choi IK, Lee JS, Zhang SN, Park J, Sonn CH, Lee KM and Yun CO: Oncolytic adenovirus co-expressing IL-12 and IL-18 improves tumor-specific immunity via differentiation of $\mathrm{T}$ cells expressing IL-12Rß2 or IL-18R $\alpha$. Gene Ther 18: 898-909, 2011.

26. Yang J, Jin G, Liu X and Liu S: Therapeutic effect of pEgr-IL18-B7.2 gene radiotherapy in B16 melanoma-bearing mice. Hum Gene Ther 18: 323-332, 2007.

27. Galon J, Costes A, Sanchez-Cabo F, Kirilovsky A, Mlecnik B, Lagorce-Pagès C, Tosolini M, Camus M, Berger A, Wind P, et al: Type, density, and location of immune cells within human colorectal tumors predict clinical outcome. Science 313: 1960-1964, 2006.
28. Sato E, Olson SH, Ahn J, Bundy B, Nishikawa H, Qian F, Jungbluth AA, Frosina D, Gnjatic S, Ambrosone C, et al: Intraepithelial CD8 tumor-infiltrating lymphocytes and a high $\mathrm{CD} 8 \mathrm{t} /$ regulatory $\mathrm{T}$ cell ratio are associated with favorable prognosis in ovarian cancer. Proc Natl Acad Sci USA 102: 18538-18543, 2005.

29. Rodrigues DR, Fernandes RK, Balderramas Hde A, Penitenti M Bachiega TF, Calvi SA, Dias-Melicio LA, Ikoma MR and Soares AM: Interferon-gamma production by human neutrophils upon stimulation by IL-12, IL-15 and IL-18 and challenge with Paracoccidioides brasiliensis. Cytokine 69: 102-109, 2014.

30. Ruiz C, Pérez E, García-Martínez O, Díaz-Rodríguez L, Arroyo-Morales M and Reyes-Botella C: Expression of cytokines IL-4, IL-12, IL-15, IL-18, and IFNgamma and modulation by different growth factors in cultured human osteoblast-like cells. J Bone Miner Metab 25: 286-292, 2007.

31. Mailliard RB, Alber SM, Shen H, Watkins SC, Kirkwood JM, Herberman RB and Kalinski P: IL-18-induced CD83+ CCR7+ NK helper cells. J Exp Med 202: 941-953, 2005.

32. Mailliard RB, Son YI, Redlinger R, Coates PT, Giermasz A, Morel PA, Storkus WJ and Kalinski P: Dendritic cells mediate NK cell help for Th1 and CTL responses: Two-signal requirement for the induction of NK cell helper function. J Immunol 171: 2366-2373, 2003.

33. Wong JL, Muthuswamy R, Bartlett DL and Kalinski P: IL-18-based combinatorial adjuvants promote the intranodal production of CCL19 by NK cells and dendritic cells of cancer patients. Oncoimmunology 2: e26245, 2013.

34. Zhong L, Roybal J, Chaerkady R, Zhang W, Choi K, Alvarez CA, Tran H, Creighton CJ, Yan S, Strieter RM, et al: Identification of secreted proteins that mediate cell-cell interactions in an in vitro model of the lung cancer microenvironment. Cancer Res 68: 7237-7245, 2008 .

35. Ribatti D, Belloni AS, Nico B, Di Comite M, Crivellato E and Vacca A: Leptin-leptin receptor are involved in angiogenesis in human hepatocellular carcinoma. Peptides 29: 1596-1602, 2008.

36. Gray-Schopfer V, Wellbrock C and Marais R: Melanoma biology and new targeted therapy. Nature 445: 851-857, 2007.

37. Yamada N, Hata M, Ohyama H, Yamanegi K, Kogoe N, Nakasho K, Futani $\mathrm{H}$, Okamura $\mathrm{H}$ and Terada $\mathrm{N}$ : Immunotherapy with interleukin-18 in combination with preoperative chemotherapy with ifosfamide effectively inhibits postoperative progression of pulmonary metastases in a mouse osteosarcoma model. Tumour Biol 30: 176-184, 2009.

38. Pappa CA, Alexandrakis MG, Boula A, Psarakis FE, Kolovou A, Bantouna V, Stavroulaki E and Tsirakis G: Emerging roles of endoglin/CD105 and angiogenic cytokines for disease development and progression in multiple myeloma patients. Hematol Oncol 31: 201-205, 2013

39. Dwivedi S, Goel A, Natu SM, Mandhani A, Khattri S and Pant KK: Diagnostic and prognostic significance of prostate specific antigen and serum interleukin 18 and 10 in patients with locally advanced prostate cancer: A prospective study. Asian Pac J Cancer Prev 12: 1843-1848, 2011.

40. Goto N, Tsurumi H, Kasahara S, Kanemura N, Hara T, Yasuda I, Shimizu M, Murakami N, Sawada M, Yamada T, et al: Serum interleukin-18 level is associated with the outcome of patients with diffuse large B-cell lymphoma treated with CHOP or R-CHOP regimens. Eur J Haematol 87: 217-227, 2011.

41. Vidal-Vanaclocha F, Mendoza L, Telleria N, Salado C, Valcárcel M, Gallot N, Carrascal T, Egilegor E, Beaskoetxea J and Dinarello CA: Clinical and experimental approaches to the pathophysiology of interleukin-18 in cancer progression. Cancer Metastasis Rev 25: 417-434, 2006.

42. Terme M, Ullrich E, Aymeric L, Meinhardt K, Desbois M, Delahaye N, Viaud S, Ryffel B, Yagita H, Kaplanski G, et al: IL-18 induces PD-1-dependent immunosuppression in cancer. Cancer Res 71: 5393-5399, 2011.

43. Terme M, Ullrich E, Aymeric L, Meinhardt K, Coudert JD, Desbois M, Ghiringhelli F, Viaud S, Ryffel B, Yagita H, et al: Cancer-induced immunosuppression: IL-18-elicited immunoablative NK cells. Cancer Res 72: 2757-2767, 2012.

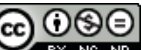

This work is licensed under a Creative Commons Attribution-NonCommercial-NoDerivatives 4.0 International (CC BY-NC-ND 4.0) License. 